\title{
Datos de la encuesta estilos de vida saludables en el contexto de la contingencia por COVID-19 en adultos-jóvenes veracruzanos
}

\author{
Survey data of healthy lifestyles in the context of the contingency \\ due to COVID-19 in young-adults from Veracruz, Mexico
}

\author{
Jorge Luis Arellanez-Hernández ${ }^{a}$ \\ León Felipe Beltrán-Guerra ${ }^{b}$ \\ Enrique Romero-Pedraza ${ }^{\mathrm{C}}$ \\ Erika Cortés-Flores ${ }^{d}$ \\ Martha Elba Ruiz-Libreros ${ }^{\mathrm{e}}$
}

Recibido: 12 de marzo de 2021.

Aceptado: 20 de abril de 2021.

RESUMEN: Se presentan resultados preliminares de la encuesta Estilo de Vida en el contexto de la contingencia por COVID-19 en adultos-jóvenes veracruzanos, utilizada en el estudio "Ansiedad, depresión, estilos de vida y bienestar en el contexto sociocultural actual". Se parte del supuesto de que el confinamiento social por la contingencia repercute de manera distinta ente hombres y mujeres. Participaron 301 personas, $76 \%$ mujeres, $24 \%$ hombres. Las mujeres reportaron un estilo

\footnotetext{
a Observatorio de Calidad de Vida y Salud Social, Instituto de Investigaciones Psicológicas, Universidad Veracruzana, México. Contacto: jarellanez@uv.mx

${ }^{b}$ Observatorio de Calidad de Vida y Salud Social, Instituto de Investigaciones Psicológicas, Universidad Veracruzana, México. Contacto: lebeltran@uv.mx

c Observatorio de Calidad de Vida y Salud Social, Instituto de Investigaciones Psicológicas, Universidad Veracruzana, México. Contacto: eromero@uv.mx

d Observatorio de Calidad de Vida y Salud Social, Instituto de Investigaciones Psicológicas, Universidad Veracruzana, México. Contacto: ecortes@uv.mx

e Observatorio de Calidad de Vida y Salud Social, Instituto de Investigaciones Psicológicas, Universidad Veracruzana, México. Contacto: maruiz@uv.mx
} 
de vida más saludable en su alimentación, actividad física, mejor estado emocional, mejores relaciones sociales, mayor interés por el cuidado de su salud física, sexual y reproductiva. Los varones, mostraron mayor consumo de alcohol, tabaco y otras drogas; y estar expuestos a ciertos riesgos de transmisión sexual o embarazos al tener más de una pareja sexual. Se deja entrever una serie de roles tradicionales de género, lo que lleva a la necesidad de analizar en estas características a mayor profundidad.

Palabras clave: estilos de vida saludables; salud mental; aislamiento social; COVID-19.

ABSTRACT: This data paper presents preliminary results of the Lifestyle survey in the context of the COVID-19 contingency in young adults from Veracruz-México, used in the study "Anxiety, depression, lifestyles and well-being in the current sociocultural context". It is based on the assumption that social confinement due to contingency affects men and women differently. 301 people participated, 76\% women, 24\% men. Women reported a healthier lifestyle in their diet, physical activity, better emotional state, better social relationships, greater interest in taking care of their physical, sexual and reproductive health. Men showed greater consumption of alcohol, tobacco and other drugs; and being exposed to certain risks of sexual transmission or pregnancy by having more than one sexual partner. A series of traditional gender roles is revealed, which leads to the need to analyze these characteristics in greater depth.

Keywords: healthy lifestyles; mental health; social isolation; COVID-19.

\section{Descripción general de los datos}

\section{Área:}

Humanidades y Ciencias de la Conducta.

\section{Sub-área:}

Psicología de la salud.

\section{Tipo de datos:}

Datos primarios, tablas.

\section{Cómo se obtuvieron los datos:}

El levantamiento de información se llevó a cabo durante el mes de junio de 2020 a través de medios digitales como el sitio Web del Observatorio de Calidad de Vida y Salud Social de la Universidad Veracruzana (OCVS), las redes sociales del OCVS (Facebook y Twitter), la cual se difundió a su vez en las redes sociales de las personas participantes en el estudio.

\section{Formato de los datos:}

Sin procesar, analizado, filtrado (estadística descriptiva e inferencial)

\section{Parámetros de la recolección de los datos:}

Participaron 301 personas entre 18 y 40 años, radicadas en el estado de Veracruz, México. 


\section{Descripción de la recolección de datos:}

Como parte de un estudio no experimental, ex post facto, con un muestreo por conveniencia, con una encuesta online se aplicó la escala "Estilo de vida" con SS ítems con un formato de respuesta tipo Likert con cinco opciones.

\section{Ubicación de la fuente de los datos:}

Veracruz, México

\section{Accesibilidad de los datos:}

En proceso.

\section{Valor de los datos}

- Los datos son importantes porque ofrecen información acerca del estilo de vida que reporta un grupo de adultos-jóvenes en el contexto del confinamiento social derivado de la contingencia por COVID-19.

- Los datos describen las seis dimensiones que permiten entender el estilo de vida de los hombres y las mujeres: alimentación, actividad física, estado emocional, relaciones sociales, consumo de alcohol, tabaco y otras drogas, y cuidado en la salud, como parte del contexto psicosocial que están viviendo por la contingencia por COVID-19. El análisis permite generar evidencia del estilo de vida matizado por las condiciones de género en condiciones contextuales específicas.

- La información reportada además de permitir identificar diferencias relacionadas con el estilo de vida entre hombres y mujeres permite poner en evidencia algunas necesidades particulares que pueden dar pie a la generación de intervenciones en grupos poblacionales con estas características considerando como eje la perspectiva de género.

\section{Descripción de los datos}

Partiendo de la concepción de salud como "un estado de completo bienestar físico, mental y social, y no solamente la ausencia de afecciones o enfermedades" (OMS, 1948:1), la pertinencia de estudios sobre el bienestar y calidad de vida en lo individual y en lo social es mayor en el contexto sociocultural actual derivado de la contingencia por COVID-19.

Las modificaciones a los comportamientos cotidianos producto del aislamiento social han generado a su vez un contexto donde los problemas sociales, de salud, económicos, incluso ambientales, han maximizado factores de riesgo que han contribuido a agudizar problemas de salud mental, particularmente en segmentos de población joven y adulto-joven. (OMS, 2019; OPS, 2020, Beltrán et. al., 2020; Ibañez et. al., 2020)

Con base en lo anterior, es que el estudio describe las características reportadas relacionadas con los estilos de vida. 


\section{Diseño Metodológico}

El estudio fue de tipo no experimental, transversal, ex post facto; y consideró un muestreo "por conveniencia", pues debido a las condiciones del confinamiento social las plataformas virtuales a través de una encuesta online han emergido como una opción pertinente para el levantamiento de información en estudios psicosociales, que además de garantizar las condiciones de sana distancia en los participantes de la investigación, son accesibles a través de distintos dispositivos electrónicos y mantienen el cuidado ético de la participación de las personas en materia de investigación en humanos.

El periodo de recolección de información comprendió el mes de junio del año 2020. La encuesta se difundió a través de las redes sociales de los participantes, partiendo de los medios digitales del Observatorio de Calidad de Vida y Salud Social: sitio Web, Facebook y Twitter.

En el presente manuscrito se reportan los hallazgos obtenidos a través de la escala Estilo de Vida, diseñada por Bazán-Riverón et al. (2019), compuesta por 40 preguntas que exploran seis áreas: alimentación (10 preguntas), actividad física (5 reactivos), estado emocional (8 ítems), relaciones sociales ( 5 preguntas), consumo de sustancias psicoactivas ( 4 reactivos) y Salud ( 8 reactivos). El formato de respuesta de la escala es de tipo Likert con cinco opciones de respuesta ( 0 a 4 ). Valores entre 0 y 1 indican un estilo de vida mórbido, el valor 2 indica un estilo de vida moderadamente saludable y entre 3 y 4 se denomina un estilo de vida saludable.

\section{Características sociodemográficas}

De las 301 personas que contestaron la totalidad del instrumento, las dos terceras partes fueron mujeres, el resto varones, con una edad promedio de 26.6 años ( $D E=6.8$ ), en un rango de 18 a 40 años; si bien cerca del $40 \%$ de los participantes se ubicaron en un rango de edad de 20 a 24 años. Alrededor del 90\% reportaron estudios universitarios.

A pesar de que el confinamiento ha acarreado una serie de limitaciones en el contexto laboral, la mayoría reportó contar con alguna ocupación, ya sea estar estudiando, contar con trabajo, o bien dedicarse a la administración del hogar, apenas un 3\% señaló no contar con alguna ocupación.

También destaca que la mayoría de las personas participantes en el estudio cuentan con acceso a servicios de salud, sobre todo de carácter público, si bien el 15\% señaló no tener acceso a este tipo de servicios. 


\section{Tabla 1. Características sociodemográficas}

\begin{tabular}{llrr} 
& & Frec & $\%$ \\
& Hombre & 71 & 23.6 \\
& Mujer & 230 & 76.4 \\
\hline \multirow{2}{*}{ Edad } & & & \\
(rangos de edad retomados de INEGI) & $15-19$ & 40 & 13.3 \\
& $20-24$ & 113 & 37.5 \\
& $25-29$ & 39 & 13.0 \\
& $30-34$ & 48 & 15.9 \\
& $35-39$ & 51 & 16.9 \\
& $40-44$ & 10 & 3.3 \\
\hline \multirow{3}{*}{ Niveles de educación } & & & \\
& Bachillerato & 32 & 10.7 \\
& Licenciatura & 235 & 78.0 \\
& Posgrado & 34 & 11.3 \\
\hline \multirow{3}{*}{ Ocupación } & & 110 & 36.5 \\
& Estudia & 119 & 39.5 \\
& Trabaja & 47 & 15.6 \\
& Estudia y trabaja & 15 & 5.0 \\
& Administración del hogar & 10 & 3.3
\end{tabular}

\section{Características sociodemográficas comparando hombres y mujeres}

La edad promedio fue ligeramente mayor entre los hombres (27 años, \pm 6.9 ) en comparación con las mujeres (26.4 años, \pm 6.8 ). Como se puede observar en la Tabla 2, el rango de edad de 20 a 24 años registra un mayor porcentaje de participación tanto en hombres como en mujeres, si bien hay una participación ligeramente mayor de mujeres de menor edad, y en los varones se registra también una participación importante en quienes reportaron una edad entre 35 y 39 años.

Destaca que las mujeres reportaron un nivel escolar ligeramente mayor en comparación con los varones, sin embargo, en estos últimos se reporta un porcentaje significativamente mayor de actividad laboral formal, así como un mayor porcentaje de encontrase sin estudiar ni trabajar. En cuanto al acceso a servicios de salud es muy parecida la información, la mayoría de los hombres y mujeres cuenta en su mayoría con acceso al sector público, alrededor del 10\% con atención en el sector privado y alrededor del 15\% no cuenta con este tipo de servicios. 
Tabla 2. Características sociodemográficas diferenciando hombres y mujeres

\begin{tabular}{llrrrr} 
& & \multicolumn{3}{c}{ Hombres } & \multicolumn{2}{c}{ Mujeres } \\
\cline { 2 - 6 } Edad & & Frec. & $\%$ & Frec. & $\%$ \\
\hline (rangos de edad retomados de INEGI) & $15-19$ & & & & \\
& $20-24$ & 4 & 5.6 & 36 & 15.7 \\
& $25-29$ & 31 & 43.7 & 82 & 35.7 \\
& $30-34$ & 9 & 12.7 & 30 & 13.0 \\
& $35-39$ & 10 & 14.1 & 38 & 16.5 \\
& $40-44$ & 15 & 21.1 & 36 & 15.7 \\
& & 2 & 2.8 & 8 & 3.5 \\
\hline \multirow{3}{*}{ Niveles de educación } & Bachillerato & & & & \\
& Licenciatura & 11 & 15.5 & 21 & 9.2 \\
& Posgrado & 56 & 78.9 & 179 & 77.8 \\
& & 4 & 5.6 & 30 & 13.0 \\
\hline \multirow{3}{*}{ Ocupación * } & Estudia & & & & \\
& Trabaja & 26 & 36.6 & 84 & 36.5 \\
& Estudia y trabaja & 31 & 43.7 & 88 & 38.3 \\
& Administración del hogar & 10 & 14.1 & 37 & 16.1 \\
& Ni estudia ni trabaja & - & - & 15 & 6.5 \\
& & 4 & 5.6 & 6 & 2.6 \\
\hline \multirow{3}{*}{ Servicios de salud } & Sin acceso & & & & \\
& Sector público & 11 & 15.5 & 34 & 14.8 \\
& Sector privado & 91 & 71.8 & 171 & 74.3 \\
& & & 12.7 & 25 & 10.9 \\
\hline
\end{tabular}

\section{Estilo de vida}

La medición del estilo de vida, entendido como la sensación de bienestar que experimenta una persona a través de su interrelación con factores personales y contextuales, debe realizarse de forma sistemática para tener la posibilidad de plantear intervenciones a grupos poblacionales con características similares, que permitan fortalecer -en la medida de lo posible-, a la persona y al grupo social con el que convive de forma cotidiana.

Como se ha señalado, el estilo de vida se midió a través de seis áreas: alimentación, actividad física, estado emocional, relaciones sociales, consumo de sustancias psicoactivas y Salud, cuyos hallazgos se presentan a continuación, diferenciando la frecuencia con la que suceden los eventos entre hombre y mujeres:

\section{- Alimentación}

En general, las mujeres muestran un estilo de vida más saludable al consumir con mayor frecuencia futas y verduras diariamente; así como una menor ingesta de azúcar en alimentos o bebidas, o de alimentos chatarra; sin embargo, reconocieron consumir con mayor frecuencia sustancias 
estimulantes como café, refrescos de cola, bebidas energéticas o medicamentos con cafeína, para tener más energía (tabla 3). Por su parte los varones reportaron un consumo más frecuente de bebidas con alto contenido de azúcar al día (chocolate, refrescos, jugos, etc.), comer más de 3 porciones de carbohidratos al día (pan, tortilla, cereales, galletas, etc.), más porciones de proteínas al día (huevo, res, cerdo, pescado, pollo, etc.), así como pedir más comida a pesar de sentirse satisfechos (tabla 3).

\section{Tabla 3. Características de la alimentación diferenciando}

\begin{tabular}{|c|c|c|c|c|c|c|c|}
\hline & Sexo & Nunca & $\begin{array}{c}\text { Casi } \\
\text { nunca }\end{array}$ & $\begin{array}{l}\text { Algunas } \\
\text { veces }\end{array}$ & $\begin{array}{l}\text { Casi } \\
\text { siempre }\end{array}$ & Siempre & M (DE) \\
\hline \multirow[t]{2}{*}{ Consumo 5 frutas y/o verduras al día } & Hombres & 11.3 & 18.3 & 50.7 & 14.1 & 5.6 & $1.85(0.99)^{*}$ \\
\hline & Mujeres & 6.5 & 20.0 & 37.0 & 26.5 & 10.0 & $2.13(1.05)$ \\
\hline \multirow{2}{*}{$\begin{array}{l}\text { A pesar de sentirme satisfecho, pido que me } \\
\text { sirvan más comida }\end{array}$} & Hombres & 23.9 & 21.1 & 46.5 & 7.0 & 1.4 & $1.41(0.97)$ \\
\hline & Mujeres & 28.7 & 30.9 & 31.7 & 7.8 & 0.9 & $1.21(0.97)$ \\
\hline \multirow{2}{*}{$\begin{array}{l}\text { Agrego más AZÚCAR de la que ya tienen mis } \\
\text { alimentos o bebidas }\end{array}$} & Hombres & 43.7 & 32.4 & 19.7 & 2.8 & 1.4 & $0.86(0.93)$ \\
\hline & Mujeres & 54.8 & 24.3 & 13.0 & 7.0 & 0.9 & $0.75(0.98)$ \\
\hline \multirow{2}{*}{$\begin{array}{l}\text { Como alimentos chatarra durante el día } \\
\text { (frituras, galletas o pan dulce) }\end{array}$} & Hombres & 4.2 & 35.2 & 47.9 & 7.0 & 5.6 & $1.75(0.87)$ \\
\hline & Mujeres & 8.7 & 32.2 & 42.6 & 13.5 & 3.0 & $1.70(0.91)$ \\
\hline \multirow{4}{*}{$\begin{array}{l}\text { Por lo menos me alimento } 3 \text { veces al día } \\
\text { (desayuno comida y cena al día) } \\
\text { Consumo más de } 3 \text { porciones de carbohidratos } \\
\text { al día (pan, tortilla, cereales, galletas, etc.) }\end{array}$} & Hombres & 1.4 & 8.5 & 22.5 & 29.6 & 38.0 & $2.94(1.0)$ \\
\hline & Mujeres & 1.7 & 9.1 & 21.3 & 29.6 & 38.3 & $2.93(1.0)$ \\
\hline & Hombres & 2.8 & 16.9 & 36.6 & 26.8 & 16.9 & $2.38(1.0)$ \\
\hline & Mujeres & 3.0 & 20.9 & 40.9 & 23.5 & 11.7 & $2.20(1.0)$ \\
\hline \multirow{2}{*}{$\begin{array}{l}\text { Consumo al menos } 3 \text { porciones de proteínas al } \\
\text { día (huevo, res, cerdo, pescado, pollo, etc.) }\end{array}$} & Hombres & - & 14.1 & 38.0 & 28.2 & 19.7 & $2.54(0.96)$ \\
\hline & Mujeres & 2.2 & 13.0 & 37.8 & 30.9 & 16.1 & $2.46(0.98)$ \\
\hline \multirow{2}{*}{$\begin{array}{l}\text { Bebo al menos } 5 \text { vasos de agua simple al día } \\
\text { (además del té, agua de frutas, refresco, café, } \\
\text { jugo, etc.) }\end{array}$} & Hombres & 1.4 & 11.3 & 19.7 & 29.6 & 38.0 & $2.92(1.07)$ \\
\hline & Mujeres & 1.7 & 12.2 & 20.9 & 28.3 & 37.0 & $2.87(1.0)$ \\
\hline \multirow{2}{*}{$\begin{array}{l}\text { Consumo más de } 1 \text { bebida con alto contenido } \\
\text { de azúcar al día (chocolate, refrescos, jugos, } \\
\text { etc.) * }\end{array}$} & Hombres & 15.5 & 36.6 & 38.0 & 7.0 & 2.8 & $1.45(0.93)$ \\
\hline & Mujeres & 19.6 & 50.4 & 20.4 & 7.8 & 1.7 & $1.22(0.90)$ \\
\hline \multirow{2}{*}{$\begin{array}{l}\text { Consumo alguna sustancia estimulante (café, } \\
\text { refrescos de cola, bebidas energéticas o } \\
\text { medicamentos con cafeína) para tener más } \\
\text { energía }\end{array}$} & Hombres & 19.7 & 22.5 & 25.4 & 19.7 & 12.7 & $1.83(1.30)$ \\
\hline & Mujeres & 23.0 & 22.2 & 28.7 & 11.3 & 14.8 & $1.73(1.33)$ \\
\hline
\end{tabular}

Nota: * p al 0.05 .

\section{- Actividad Física}

En cuanto al estilo de vida por actividad física las mujeres también registraron características más saludables, con una frecuencia significativamente más alta que los hombres reportaron practicar algún deporte, de bajo riesgo, aprovechar el tiempo libre en leer o realizar alguna actividad artística, así como usar con menor frecuencia las redes sociales digitales, ver la televisión o usar videojuegos. En contraparte, los varones registraron con mayor frecuencia actividades predominantemente sedentarias o actividades deportivas de alto riesgo, como usar patineta, hacer parkour, por ejemplo. 
Tabla 4. Características de la actividad física diferenciando hombres y mujeres

\begin{tabular}{|c|c|c|c|c|c|c|c|}
\hline & Sexo & Nunca & $\begin{array}{c}\text { Casi } \\
\text { nunca }\end{array}$ & $\begin{array}{l}\text { Algunas } \\
\text { veces }\end{array}$ & $\begin{array}{l}\text { Casi } \\
\text { siempre }\end{array}$ & Siempre & M (DE) \\
\hline Cuando estoy en la escuela, hago activación & Hombres & 9.9 & 26.8 & 26.8 & 19.7 & 16.9 & $2.07(1.24)$ \\
\hline física, más de 3 veces a la semana & Mujeres & 17.0 & 22.6 & 21.3 & 19.6 & 19.6 & $2.02(1.37)$ \\
\hline Practico algún deporte en mi tiempo libre & Hombres & 28.2 & 29.6 & 21.1 & 15.5 & 5.6 & $1.41(1.21)^{* *}$ \\
\hline (natación, futbol, danza, etc.) * & Mujeres & 51.7 & 19.1 & 9.1 & 11.7 & 8.3 & $1.06(1.35)$ \\
\hline En mi tiempo libre, suelo revisar mis redes & Hombres & - & 4.2 & 33.8 & 28.2 & 33.8 & $2.92(0.92) * *$ \\
\hline $\begin{array}{l}\text { sociales, ver televisión o jugar videojuegos más } \\
\text { de } 3 \text { horas diarias ** }\end{array}$ & Mujeres & 3.5 & 10.9 & 31.3 & 33.5 & 20.9 & $2.57(1.04)$ \\
\hline En mi tiempo libre, suelo leer o realizar alguna & Hombres & 11.3 & 36.6 & 31.0 & 16.9 & 4.2 & $1.66(1.02)$ \\
\hline $\begin{array}{l}\text { actividad artística (pintar, dibujar, tocar algún } \\
\text { instrumento, etc.) }\end{array}$ & Mujeres & 13.9 & 21.3 & 31.7 & 25.2 & 7.8 & $1.92(1.15)$ \\
\hline Realizo algún deporte de alto riesgo (patineta, & Hombres & 71.8 & 16.9 & 8.5 & 1.4 & 1.4 & $0.44(0.82)^{* *}$ \\
\hline $\begin{array}{l}\text { parkour -Trepar y saltar por muros haciendo } \\
\text { acrobacias-, otro) * }\end{array}$ & Mujeres & 87.4 & 8.7 & 1.3 & 1.7 & 0.9 & $0.20(0.63)$ \\
\hline
\end{tabular}

Nota: $* \mathrm{p}$ al $0.01{ }^{* *} \mathrm{p}$ al 0.05 .

\section{- Estado Emocional}

Las mujeres reportan un estado emocional un tanto más saludable en comparación con los varones, pues reportan sentirse atractivas, y con facilidad para expresar sus sentimientos; sin embargo, las semanas previas a la encuesta se percibieron nerviosas o estresadas. Por su parte, los varones reportan un estado emocional un tanto menos saludable en comparación con las mujeres, pues reportaron que con mayor frecuencia se sienten menos reconocidos o valorados por los demás, sentir que en el futuro nada les saldrá bien, así como enojarse con facilidad y haberse sentido enfermos y/o sin energía los 30 previos a su participación en el estudio. Particularmente sobresale que tanto hombres como mujeres reportan una alta frecuencia de "enojo con facilidad", lo que sugiere cierta sensibilidad emocional en la vida cotidiana.

Tabla 5. Características del estado emocional diferenciando hombres y mujeres

\begin{tabular}{|c|c|c|c|c|c|c|c|}
\hline & Sexo & Nunca & $\begin{array}{c}\text { Casi } \\
\text { nunca }\end{array}$ & $\begin{array}{l}\text { Algunas } \\
\text { veces }\end{array}$ & $\begin{array}{l}\text { Casi } \\
\text { siempre }\end{array}$ & Siempre & M (DE) \\
\hline \multirow[t]{2}{*}{ Me enojo con facilidad } & Hombres & 2.8 & 23.9 & 38.0 & 28.2 & 7.0 & $2.13(0.95)$ \\
\hline & Mujeres & 5.2 & 17.4 & 47.8 & 20.0 & 9.6 & $2.11(0.97)$ \\
\hline \multirow{2}{*}{$\begin{array}{l}\text { Tengo cambios repentinos de humor (de triste a } \\
\text { feliz, de feliz a enojado, etc.) }\end{array}$} & Hombres & 14.1 & 22.5 & 36.6 & 18.3 & 8.5 & $1.85(1.14)$ \\
\hline & Mujeres & 15.7 & 25.2 & 32.2 & 18.7 & 8.3 & $1.79(1.16)$ \\
\hline \multirow{2}{*}{$\begin{array}{l}\text { Me he sentido enfermo y/o sin energía en las } \\
\text { últimas semanas }\end{array}$} & Hombres & 11.3 & 23.9 & 29.6 & 21.1 & 14.1 & $2.03(1.21)$ \\
\hline & Mujeres & 13.0 & 16.1 & 38.7 & 22.2 & 10.0 & $2.00(1.14)$ \\
\hline \multirow{2}{*}{$\begin{array}{l}\text { Siento que mis habilidades no son reconocidas o } \\
\text { valoradas como quisiera* }\end{array}$} & Hombres & 5.6 & 16.9 & 45.1 & 16.9 & 15.5 & $2.20(1.07)^{* *}$ \\
\hline & Mujeres & 18.3 & 19.1 & 33.0 & 21.7 & 7.8 & $1.82(1.19)$ \\
\hline \multirow{2}{*}{$\begin{array}{l}\text { Suelo pensar que en el futuro nada me saldrá } \\
\text { bien ni de la forma que yo quiero }\end{array}$} & Hombres & 25.4 & 22.5 & 28.2 & 12.7 & 11.3 & $1.62(1.30)$ \\
\hline & Mujeres & 27.8 & 27.0 & 24.3 & 12.6 & 8.3 & $1.47(1.24)$ \\
\hline \multirow{2}{*}{$\begin{array}{l}\text { Me he sentido tenso, nervioso o estresado en las } \\
\text { últimas semanas }\end{array}$} & Hombres & 5.6 & 14.1 & 33.8 & 28.2 & 18.3 & $2.39(1.11)$ \\
\hline & Mujeres & 4.3 & 13.0 & 32.2 & 27.4 & 23.0 & $2.52(1.11)$ \\
\hline \multirow[t]{2}{*}{ Soy atractivo(a) para los demás } & Hombres & 12.7 & 14.1 & 45.1 & 23.9 & 4.2 & $1.93(1.03)$ \\
\hline & Mujeres & 5.7 & 15.2 & 42.6 & 31.3 & 5.2 & $2.15(0.93)$ \\
\hline \multirow[t]{2}{*}{ Expreso mis sentimientos con facilidad } & Hombres & 11.3 & 25.4 & 36.6 & 11.3 & 15.5 & $1.94(1.20)$ \\
\hline & Mujeres & 10.4 & 20.4 & 31.7 & 23.9 & 13.5 & $2.10(1.18)$ \\
\hline
\end{tabular}

Nota: ${ }^{*} \mathrm{p}$ al $0.01 ;{ }^{* *} \mathrm{p}$ al 0.05 . 


\section{- Relaciones Sociales}

Con respecto a el establecimiento de redes sociales de apoyo, las mujeres registraron un apoyo significativamente mayor por parte de sus familiares, así como de sus amigos y compañeros, que les genera satisfacción; sin embargo, también reportaron mayores episodios de algún tipo de violencia en su casa o en la escuela (gritos, insultos, burlas, golpes).

Tabla 6. Características de relaciones sociales diferenciando hombres y mujeres

\begin{tabular}{|c|c|c|c|c|c|c|c|}
\hline & Sexo & Nunca & $\begin{array}{c}\text { Casi } \\
\text { nunca }\end{array}$ & $\begin{array}{l}\text { Algunas } \\
\text { veces }\end{array}$ & $\begin{array}{c}\text { Casi } \\
\text { siempre }\end{array}$ & Siempre & $\bar{M}(\mathrm{DE})$ \\
\hline \multirow{4}{*}{$\begin{array}{l}\text { Cuando tengo algún problema, recurro a mi } \\
\text { familia * } \\
\text { Mi familia se interesa por mi bienestar físico }\end{array}$} & Hombres & 14.1 & 28.2 & 33.8 & 16.9 & 7.0 & $1.75(1.11)^{* *}$ \\
\hline & Mujeres & 6.5 & 21.3 & 28.3 & 27.0 & 17.0 & $2.27(1.16)$ \\
\hline & Hombres & 5.6 & 11.3 & 22.5 & 29.6 & 31.0 & $2.69(1.19) *$ \\
\hline & Mujeres & 3.9 & 5.2 & 22.6 & 23.9 & 44.3 & $3.00(1.11)$ \\
\hline \multirow{4}{*}{$\begin{array}{l}\text { Siento que mi familia me apoya en las } \\
\text { decisiones que tomo } \\
\text { Estoy satisfecho con mis relaciones sociales con } \\
\text { amigos, compañeros y profesores }\end{array}$} & Hombres & 5.6 & 9.9 & 26.8 & 28.2 & 29.6 & $2.66(1.17)$ \\
\hline & Mujeres & 3.9 & 7.8 & 27.0 & 30.0 & 31.3 & 2.77 (1.09) \\
\hline & Hombres & 4.2 & 5.6 & 33.8 & 39.4 & 16.9 & $2.59(0.97)$ \\
\hline & Mujeres & 2.2 & 7.8 & 25.7 & 35.2 & 29.1 & $2.81(1.01)$ \\
\hline \multirow{2}{*}{$\begin{array}{l}\text { Sufro algún tipo de violencia en mi casa o en la } \\
\text { escuela (gritos, insultos, burlas, golpes) }{ }^{* *}\end{array}$} & Hombres & 60.6 & 29.6 & 8.5 & 1.4 & - & $0.51(0.75)$ \\
\hline & Mujeres & 77.8 & 10.9 & 7.8 & 2.2 & 1.3 & $0.38(0.82)$ \\
\hline
\end{tabular}

Nota: *p al $0.05 ; * *$ p al 0.01 .

\section{— Salud (consumo de alcohol, tabaco y otras drogas)}

Es de destacar que el estilo de vida de las mujeres en cuanto al consumo de sustancias psicoactivas como alcohol, tabaco u otras drogas es significativamente mayor al de los varones. Si bien se llega a reportar el uso de estas sustancias en algunas mujeres, en general es mucho menor al que reportan los varones. En particular sobresale la frecuencia del consumo en alcohol y tabaco que registran ciertas similitudes entre hombres y mujeres.

\begin{tabular}{|c|c|c|c|c|c|c|c|}
\hline \multicolumn{8}{|c|}{$\begin{array}{r}\text { Tabla 7. Características de consumo de alcohol, taba } \\
\text { hombres y mujeres }\end{array}$} \\
\hline & Sexo & Nunca & $\begin{array}{c}\text { Casi } \\
\text { nunca }\end{array}$ & $\begin{array}{c}\text { Algunas } \\
\text { veces }\end{array}$ & $\begin{array}{c}\text { Casi } \\
\text { siempre }\end{array}$ & Siempre & $\mathrm{M}(\mathrm{DE})$ \\
\hline \multirow{2}{*}{$\begin{array}{l}\text { En fiestas tomo más de } 2 \text { bebidas con alcohol } \\
\text { (como vasos de cerveza, cubas, bebidas } \\
\text { preparadas) }\end{array}$} & Hombres & 9.9 & 11.3 & 29.6 & 28.2 & 21.1 & $2.39(1.22)^{* *}$ \\
\hline & Mujeres & 26.5 & 14.8 & 28.3 & 18.7 & 11.7 & $1.74(1.34)$ \\
\hline \multirow[t]{2}{*}{ Fumo $* *$} & Hombres & 40.8 & 19.7 & 22.5 & 2.8 & 14.1 & $1.30(1.39)^{* *}$ \\
\hline & Mujeres & 64.3 & 11.3 & 17.4 & 3.9 & 3.0 & 0.70 (1.07) \\
\hline \multirow{2}{*}{$\begin{array}{l}\text { Consumo bebidas alcohólicas más de } 1 \text { vez a la } \\
\text { semana ** }\end{array}$} & Hombres & 26.8 & 38.0 & 23.9 & 8.5 & 2.8 & $1.23(1.03)^{* *}$ \\
\hline & Mujeres & 51.7 & 28.7 & 13.0 & 6.1 & 0.4 & $0.75(0.93)$ \\
\hline \multirow{2}{*}{$\begin{array}{l}\text { Consumo drogas (marihuana, cocaína, } \\
\text { inhalantes, cristal, heroína, etc.) ** }\end{array}$} & Hombres & 69.0 & 31.0 & - & - & - & $0.31(0.46)^{* *}$ \\
\hline & Mujeres & 91.3 & 8.7 & - & - & - & $0.09(0.28)$ \\
\hline
\end{tabular}

Nota: ${ }^{*} \mathrm{p}$ al $0.05 ; * * \mathrm{p}$ al 0.01 


\section{— Salud (cuidado preventivo)}

Al igual que en las otras áreas, las mujeres también registraron mayor cuidado de su salud en cuanto a dormir lo necesario, realizarse chequeos médicos preventivos y de tomar medidas en general de su salud para en la medida de lo posible, evitar algunas enfermedades.

\begin{tabular}{|c|c|c|c|c|c|c|c|}
\hline & Sexo & Nunca & $\begin{array}{c}\text { Casi } \\
\text { nunca }\end{array}$ & $\begin{array}{l}\text { Algunas } \\
\text { veces }\end{array}$ & $\begin{array}{c}\text { Casi } \\
\text { siempre }\end{array}$ & Siempre & $\mathrm{M}(\mathrm{DE})$ \\
\hline \multirow{2}{*}{$\begin{array}{l}\text { Estando sano, asisto a realizarme chequeos } \\
\text { médicos preventivos (con médico familiar o } \\
\text { pediatra, dentista, optometrista, etc.) }{ }^{* *}\end{array}$} & Hombres & 39.4 & 21.1 & 31.0 & 7.0 & 1.4 & $1.10(1.05)^{* *}$ \\
\hline & Mujeres & 20.4 & 26.1 & 33.0 & 14.8 & 5.7 & $1.59(1.13)$ \\
\hline \multirow{2}{*}{$\begin{array}{l}\text { En mi familia tomamos medidas generales de } \\
\text { salud (desparasitación, vacunación, etc.) } \\
* *\end{array}$} & Hombres & 7.0 & 22.5 & 33.8 & 19.7 & 16.9 & $2.17(1.17)^{* *}$ \\
\hline & Mujeres & 3.0 & 10.0 & 30.0 & 28.3 & 28.7 & $2.70(1.08)$ \\
\hline \multirow[t]{2}{*}{ Suelo dormir menos de 7 horas diariamente } & Hombres & 4.2 & 29.6 & 32.4 & 26.8 & 7.0 & $2.03(1.01)$ \\
\hline & Mujeres & 7.8 & 20.0 & 38.7 & 22.6 & 10.9 & $2.09(1.08)$ \\
\hline \multirow{2}{*}{$\begin{array}{l}\text { Consumo proteínas en polvo, anfetaminas o } \\
\text { medicamentos para controlar mi peso }\end{array}$} & Hombres & 81.7 & 7.0 & 8.5 & 2.8 & - & $0.32(0.75)$ \\
\hline & Mujeres & 77.4 & 8.7 & 8.7 & 3.9 & 1.3 & $0.43(0.90)$ \\
\hline \multirow{2}{*}{$\begin{array}{l}\text { Consulto con mis maestros y/o padres } \\
\text { información acerca de métodos } \\
\text { anticonceptivos y enfermedades de } \\
\text { transmisión sexual (ETS) }\end{array}$} & Hombres & 15.5 & 15.5 & 36.6 & 18.3 & 14.1 & $2.00(1.24)$ \\
\hline & Mujeres & 12.6 & 12.2 & 33.5 & 17.0 & 24.8 & $2.29(1.30)$ \\
\hline
\end{tabular}

Nota: ${ }^{*} \mathrm{p}$ al $0.05 ;{ }^{* *} \mathrm{p}$ al 0.01

\section{— Salud (cuidado de la vida sexual)}

Con respecto al comportamiento en el "cuidado en la vida sexual", al igual que en las otras áreas de salud, las mujeres recurren con mayor frecuencia a métodos anticonceptivos, en comparación con los varones y tienen parejas sexuales relativamente más estables.

\begin{tabular}{|c|c|c|c|c|c|c|c|}
\hline & Sexo & Nunca & $\begin{array}{c}\text { Casi } \\
\text { nunca }\end{array}$ & $\begin{array}{c}\text { Algunas } \\
\text { veces }\end{array}$ & $\begin{array}{c}\text { Casi } \\
\text { siempre }\end{array}$ & Siempre & M (DE) \\
\hline \multirow{2}{*}{$\begin{array}{l}\text { Utilizo algún método anticonceptivo al tener } \\
\text { relaciones sexuales }\end{array}$} & Hombres & 19.7 & 11.3 & 21.1 & 19.7 & 28.2 & $2.25(1.48)$ \\
\hline & Mujeres & 23.5 & 5.7 & 13.0 & 17.4 & 40.4 & $2.46(1.60)$ \\
\hline \multirow[t]{2}{*}{ Utilizo CONDÓN en mis relaciones sexuales } & Hombres & 16.9 & 18.3 & 19.7 & 23.9 & 21.1 & $2.14(1.39)$ \\
\hline & Mujeres & 27.0 & 10.4 & 15.7 & 17.8 & 29.1 & $2.12(1.58)$ \\
\hline \multirow[t]{2}{*}{ Tengo más de una pareja sexual ** } & Hombres & 52.1 & 15.5 & 19.7 & 5.6 & 7.0 & $1.00(1.26)^{* *}$ \\
\hline & Mujeres & 81.7 & 9.6 & 7.0 & 0.9 & 0.9 & $0.30(0.71)$ \\
\hline
\end{tabular}

Nota: *p al $0.05 ;{ }^{* *} \mathrm{p}$ al 0.01 


\section{Consideraciones éticas}

La encuesta cuidó tomar en cuenta las normas éticas de la investigación en seres humanos, tomando como elemento central el cuidado del anonimato y la confidencialidad a través del consentimiento informado (Secretaría de Salud 1983, 2013; INSP, 2016).

Declaración de conflicto de intereses

Los autores declaran no tener ningún conflicto de intereses potencial con respecto a la investigación, autoría y / o publicación de este artículo.

Financiamiento

Los autores declaran no haber recibido financiamiento para la investigación, autoría y / o publicación de este artículo.

\section{Referencias}

Bazán-Riverón, G.E., Osorio-Guzmán, M., Torres-Velázquez, L.E., Rodríguez-Martínez, J.I., Ocampo-Jasso, J.A. (2019). Validación de una escala sobre estilo de vida para adolescentes mexicanos. Revista Mexicana de Pediatría; 86(3):112-118.

Beltrán-Guerra, L.F., Arellanez-Hernández, J.L. y Romero Pedraza, E. (2020). Medición del Bienestar Subjetivo y Condiciones de Vida en el contexto sociocultural de la contingencia por COVID en Veracruz, México. UVserva, (10,) 94-103.

Instituto Nacional de Salud Pública (INSP) (2016). Consentimiento informado. http://www.insp.mx/insp-cei/consentimiento-informado.html

Ibañez-Vizoso, J. E., Alberdi-Páramo, I., Díaz-Marsá, M. (2020). International Mental Health perspectives on the novel coronavirus SARS-CoV-2 pandemic. Revista Psiquiátrica de Salud Mental, 13:109-111.

Organización Mundial de la Salud (OMS). (2019). Salud mental del adolescente. https://bit.ly/3dEmpAc

Organización Panamericana de la Salud (OPS). (2020). Informe COVID 19 CEPAL-OPS. Salud y economía: una convergencia necesaria para enfrentar el COVID-19 y retomar la senda hacia el desarrollo sostenible en América Latina y el Caribe. https://bit.ly/32Gzrqd

Organización Mundial de la Salud (1948). Constitución de la Organización Mundial de la Salud. https://bit.ly/3xdXsDj

Secretaría de Salud (1983). Reglamento de la Ley General de Salud en Materia de Investigación para la Salud. Diario Oficial de la Federación. Consultado el 12 de julio de 2019. https://bit.ly/3xhlpK6

Secretaría de Salud (2013). NORMA Oficial Mexicana NOM-012-SSA3-2012, Que establece los criterios para la ejecución de proyectos de investigación para la salud en seres humanos. Diario Oficial de la Federación. Consultado el 09 de mayo de 2019. https://bit.ly/3sEbZ7F 